\title{
Anticancer Drug Development, Getting Out from Bottleneck
}

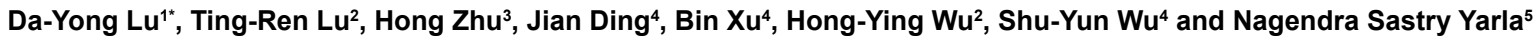 \\ ${ }^{1}$ School of Life Sciences, Shanghai University, Shanghai, PR China \\ ${ }^{2}$ College of Science, Shanghai University, Shanghai, PR China \\ ${ }^{3}$ Zhejiang University, Hangzhou, PR China \\ ${ }^{4}$ Shanghai Institute of Materia Medica, Chinese Academy of Sciences, Shanghai, PR China \\ ${ }^{5}$ GITAM University, Vishakhapatnam, Andra Pradesh, India
}

\begin{abstract}
Drug development as a sensitive thermometer represents the scientific and technical power-engines for any countries. As a result, drug manufacture (highly competitive area) is pillar industry for small-range of developed countries and highest medical expenditures worldwide. Nonetheless, new anticancer drug discovery, development and manufacture were entering into bottleneck stage two decades ago. A declining successful rate of phase II and phase III anticancer drug evaluations is the grimmest situation for most leading anticancer drug developers worldwide. Profit is down every years. Owing to all these kinds of undesired factors, anticancer drug developments manufacturing and licensing have been highly risky over the past two decades. This article addresses key factors affecting anticancer drug developments and possible roadmaps for drug screening model and routine updating in all relevant countries are especially speculated.
\end{abstract}

Keywords: Anticancer drug development; Neoplasm metastasis; Cost-effective; Medicinal chemistry; Animal models; Drug toxicity; Cancer stem cells; Individualized cancer therapy

\section{Introduction}

\section{Background}

Cancer is a malignant disease that costs 7-10 million human mortalities annually worldwide [1,2]. Despite great advances, current cancer therapy has still many limitations, e.g., high costs for conventional therapy and shortage of effective anticancer and antimetastatic drugs for late-staged cancer patients [3-7]. For this circumstance, great changes in the fields of cancer therapeutic studies are disparately needed.

\section{Current situation in cancer therapeutics and drug developments}

Drug manufactory is a pillar industry for small number of worldleading countries, which is a highly competitive and risky job worldwide [8-11]. Nevertheless drug discovery, development and manufacture have been entering into bottleneck stages since two decade ago-productivity and successful rates of clinical drug evaluation was declining year-byyear [11]. Profit for big manufactures is down. Despite greater fortune spent for the whole process of each anticancer licensing (1-2 billion USD) in US and other developed countries, [11-13] cancer therapies improved slightly and are still imperfect in clinical practice, especially for cancer metastasis treatments [3-7,14-22]. Thus anticancer drug discoveries and developments are highly risky processes and negative impacts for healthy progresses of drug development and manufacturing worldwide [4-13].

\section{Anticancer drug development dilemma}

- Growing number of modern biological techniques and systems has been developed. In order to use and evaluate drug therapeutic efficacies and toxicities by these modern biological techniques and systems, much more money has to be paid off.

- Since many biological or pathological properties of cancers, such as neoplasm metastasis and cancer stem cells have not been well understood, new anticancer drugs have to be produced from random experimental screening and clinical toxicity evaluations. This is also very expensive and low-efficiency.

- Current policy of anticancer drug licensing is that new compounds must be more effective than licensed anticancer drugs. This is a paradox issue because cancer is a different disease that needs to be targeted or treated by different anticancer drugs. Thus rigid drug evaluation and regulatory rule forbid healthy progresses of anticancer drug developments and manufacture [10].

\section{Results}

Scenarios and keys of current anticancer drug discoveries and developments

Great diversity of cancer models: Anticancer drugs are divided into wide-spectra and narrow-spectra that target on different types of cancer genes, biological molecules and mechanisms of actions. They often act onto different types of animal or human tumor models and pathophysiological pathways in clinics. Similarly, cancer is different diseases that share the common pathologic characters of unlimited growths. Clinically, >100 tumor types can be found in clinics [23]. Facing this requirement, future experimental or preclinical tumor models should be categorized into different series and be screened by different types of anticancer agents and combinations. Present in vitro or in vivo drug screening animal or human tumor models are enormously diversified in major drug developed countries [8-10]. For example, approximately 1,200 tumor cell lines are stored for anticancer drug screening, verifications and mechanism explorations in America Tissue Culture and Collection (ATCC), USA. As a result, proper budget control systems must be established. Yet most of these animal or human tumor models have lost their original genotypes or phenotypes by long passages in vitro or in vivo, which make relatively low quality of preclinical drug activity studies. The good balance between model

*Corresponding authors: Da-Yong Lu, School of Life Sciences, Shanghai University, Shanghai 200444, PR China, Tel: +862166163545; Fax: +862166132177; E-mail: ludayong@shu.edu.cn

Received January 05, 2017; Accepted January 19, 2017; Published January 23 2017

Citation: Lu D, Lu T, Zhu H, Ding J, Xu B, et al. (2017) Anticancer Drug Development, Getting Out from Bottleneck. Med Chem (Los Angeles) 7: 739-744. doi: 10.4172/2161-0444.1000423

Copyright: @ 2017 Lu D, et al. This is an open-access article distributed under the terms of the Creative Commons Attribution License, which permits unrestricted use, distribution, and reproduction in any medium, provided the original author and source are credited. 
diversity and mechanism similarity is a hotspot for drug developments and industry competent.

Pathologic and pharmacologic characters: Different tumor inoculation routes or final evaluating systems in experimental or preclinical studies may also affect new compound responses/efficacy outcomes to tumor models and pharmacological data statistically analysis. Initially, in vivo tumor models were mostly inoculated unto subcutaneous locations (sc), intraperatoneal (ip), intravenous (iv) and lately hollow-fiber from ectopic tumor origins or xenografts for new compound anticancer or antimetastatic activity testing. More recently, transplantations of human tumor xenografts into immune-deficient animals are available for expanding experimental conditions to clinical therapeutic circumstances. In additionally, tumors may be inoculated into orthotopic sites or genetic engineering mice models (GEMM). Similarly, environmental factors or surroundings can aid tumor tissues originally survival and progresses in animal or human bodies and test different types of anticancer drug targets. With these experimental advancements, more effective anticancer drugs are proposed to be found.

Tumor metastasis experimental/clinic models and specific targeted drugs: Antimetastatic agents or drugs developments need to be boosted and promoted with times because $90 \%$ cancer patient mortalities are caused by neoplasm metastasis, especially for aged cancer patients. Despite steady increases of biology and pathology knowledge towards neoplasm metastasis, their therapeutic efficacy and mechanisms of action by antimetastatic drugs are still not overwhelmed in the past three decades even with state-of-the-art technologies. Few antimetastatic agent or drug exhibits high therapeutic efficacies in cancer patients with neoplasm metastases [3-7,14-22]. Even though widely agreed as a top priority and future trend, currently experimental tumor metastatic models are insufficient for successfully harvesting enough effective antimetastatic drugs. Shortage of wide-spectra and highly active antimetastatic drugs is a serious problem for clinical utilities and therapeutic responses for late-staged and aged cancer patients.

\section{Discussion}

\section{New insights into anticancer drug screening models and lab equipments}

Anticancer drug screening models updating for different types of therapeutic purposes is a key issue in anticancer drug developments and clinical applications [11-13]. Though new generations of in vitro or in vivo tumor models have been invented, the overall outlook of anticancer drug development systems changed slightly. It means we did not hit the bull's eye of anticancer drug developments in the past. We have to adapt all smart and practical ideas (such as angiogenesis or cancer stem cell inhibitors) in drug developmental processes even though these pathogenesis processes represent only small parts of neoplasm pathogenesis progresses, invasive and remote metastases. Yet any small pieces of biological or pathogenesis information may finally help us to jigsaw a wholesome picture of cancer that effective therapeutic interventions are then originated. Overall, it is not the time to debate or nullify previously discovered processes. It is the time to integrate and jigsaw these pieces and puzzle altogether.

\section{Updating anticancer drug screening systems}

There are approximately 1,200 human cancer cell lines in conventional drug screening systems in ATCC, US. However, these enormous types of human cell lines share a lot of biological similarity and equivalent pathologic pathways after long passage of tumor cells in vitro conditions. If we keep on previous work, a lot of money will be paid in vain. Three avenues can be undertaken to improve this setback.

- Categorize these 1,200 human tumor cell lines into several groups according to their biologic, pathologic and therapeutic characteristics $[8,9]$. Several tumor cell lines with obvious unique properties of specific tumor characteristics are then used for drug screenings at utilities of least amounts of tumor cell lines; Endless efforts can be applied to this single topic. The mature experimental models in this study, the more usefulness can be expected for anticancer drug discoveries and promotions;

- Genetic- or other modern-techniques modified tumor types and models may be introduced and these modified tumor models may be more parallel to clinical situations and therapeutic outcomes than previous ones;

- Since clinical drug evaluations are costly and inconvenience and indispensable parts of drug developments. Facing this dilemma, utilities of clinical tumor banks for preclinical drug evaluations and assessments [24] can be an alternative solution.

\section{Avant-garde experimental equipments and lab facilities}

Apart from animal or human tumor models, avant-garde experimental equipments and lab facilities can also improve the drug evaluation qualities of drug therapeutic efficacies and toxicity identifications. Here are the several examples that a modern lab may require and be equipped;

\section{Some examples of cutting-edge lab devises for drug} evaluations and assessments

- Electric tumor volume detectors

- Magnetic resonance imaging (MRI)

- Positron emission tomography

- Bioluminescent imaging

- Modern chromatography (HPLC-MS, GC-IR, GC-MS, etc.)

- Next generation sequencing machine

- Tumor biomarker detection (Automatic Western blot devise, etc.)

- Different types of high-throughput bioinformatics techniques and equipments

Despite growing variety of available animal or human tumor models, avant-garde equipments and facility improvements of anticancer drug discovery pipelines in modern era. Nonetheless advancements of new anticancer drug discovery by this avenue have been slow in pace while the cost of anticancer drug developments is greatly heightened. By entering into this Millennium, initial drug screening process and clinical verification fee is soaring after these technical inventions and instrument utilities. Strangely enough, these advancements of tumor models and automatic detection equipments commonly only help us to reclaim a number of withdrawal anticancer drugs instead of finding novel anticancer drugs $[12,25]$. The causations behind this scenery are important fields of anticancer drug developments and up-to-date licensing systems. 


\section{Anticancer drug developments, a matter of money or a matter of ideas}

Anticancer drug developments, a matter of money or a matter of ideas is an important question to be resolved [10]. From abovementioned topics, greater parts of arguments are addressing on the importance of reducing unnecessary drug development costs. Similarly, scientific investigations about fundamental areas of pharmacology and therapeutics and recruiting high talented personnel are no less importance than experimental models or cutting-edge technique utilities [10]. Money is always easier to collect than marvelous persons. But it is the indispensable in initial stages of dramatic changes and high talented person recruiting. Not only for high talented medicinal chemists, pharmacologists and clinical doctors, but also for joining hands with mathematic/physics-majored students and scholars might be sought after worldwide [26,27]. These kinds of mathematic/physicsmajored students and scholars ought not only to play assistant roles as usual ways, but also change the landscapes of anticancer drug developments and marketing [26].

\section{Good governmental policy supports for drug development}

Good governmental regulatory policy supports might stimulate anticancer drug development and establish efficiency drug screening systems globally. Many median-sized industrial and BRICS should not satisfy only with publishing articles in high impact factor international journals, their ambitions for drug developments also play important roles for world healthcare advancements.

\section{To evaluate the efficacy of different drug combinations}

Cancer metastasis is a malignant phenotype that is often difficult to be completely managed. Despite great advances, current cancer therapy has limitations in many respective, such as high costs of conventional therapy, shortages of highly effective antimetastatic drugs and so on [3-10]. To overcome these obstacles, anticancer drug combination is a useful way to improve therapeutic outcomes in clinical cancer trials [28].

Evaluating therapeutic responses of different drug combinations is a new hotspot for anticancer drug studies. After systematic scientific efforts, anticancer drug combinations can be translated from benches to the bedsides. Previously, large volumes of drug combinative study are discovered directly from clinical cancer therapeutics.. To enhance this clinical enterprise, new drug screening systems must be established $[29,30]$.

\section{Pharmaceutical study of anticancer drug delivery systems}

Pharmaceutical study of anticancer drug delivery systems is an unavoidable avenue to improve cancer therapeutics. Different pharmaceutical forms of anticancer drugs may vary in therapeutic efficacy against primary tumor growth and remote disseminations. May the nano anticancer drug, an emerging topic of cancer therapeutic approach make a difference in clinical trials? A great number of references support this scenario and want to promote nano-technology into better anticancer drug therapeutics [31,32]. Yet, some of these references exaggerate their therapeutic efficacy and clinical applications due to higher immune-system toxicities of nano-particle anticancer drug treatments. If we pay no heed to these exaggerations, we will stay at original sites for long times. Scientifically analysis this topic in noteworthy.

\section{Personalized cancer therapies in clinics}

Apart from finding enough active anticancer or antimetastatic drugs, good predictive models of drug responsive in clinics can also improve therapeutic efficacy - including drug sensitivity testing [3335], pharmacogenetics (PGx) [36-39], cancer bioinformatics and individualized antimetastatic therapies [40-44] and so on. These types of clinical efforts are called personalized cancer therapy (PCT) or individualized cancer therapy (ICT) [44]. Rapid advancements of these disciplines are 'high profile' field in clinical trials. In the future, increasing events of PCT might be mandatory routines in clinical cancer practices in developed countries, even widely utilities worldwide.

\section{Importance of future drug development systems}

The future drug development systems can be outlined in Figure 1. With the completeness of these systems, anticancer drug developments might be get out from bottleneck.

\section{Hot Topics}

Apart from above-mentioned topics, a number of recent hotspots of anticancer drug developments are emphasized herein.

Key factors affecting the quality of experimental, preclinical and clinical anticancer drug evaluations

Several steps of optimal compound combinations, dose ranges, administered schedules and possible therapeutic durations in animal models and in humans are theoretically very difficult. The discovery of drug targets and mechanisms of action of new compounds can establish the relationship between cancer diagnostics and therapeutic benefits/ toxicities. However, cancer is diversity diseases with different oncology origin and promoting processes started from various genetic changes in normal cells. It could be $>100$ different cancer types in clinics [23]. As a result, picking up effective compounds from great chemical pipelines is the key for making experimental, preclinical and clinical drug evaluation updating.

Several steps for improving drug screening protocols and systems are categorized into following interesting topics. Several important obstacles must be overcome, including;

- As usual, simultaneously screen multiple targets (genotypic or phenotypic-related), gene-mutation, tumor suppressor losses, signaling-pathway abnormalities and drug responses/risks in evaluated tumor cell lines and solid tumors must be focused. To optimally use those animal or human tumor models can reduce money and promote efficiency of anticancer drug screening.

- Efforts must be made to reduce anticancer drug evaluations and development cost. It would benefit cancer patients from modern science and technology utilities in order to save more cancer patients. Drug developmental budget and cost-effective considerations in clinics should be optimally controlled worldwide $[45,46]$.

- Since the scientific study of the relationship between cancer biology, pathology and therapeutics is the fundamental areas of drug developments, future efforts on this matter will be the top priority.

- Implement high quality drug tolerability and PD/PK study in both animals and humans must be encouraged, which are important building-blocks of anticancer drug development [36-39]. Nevertheless, this is an endless enterprise that can be varied among different individuals. Rational design these work and standardize basic toxicity evaluating systems/protocols in clinical circumstances needs to be well established.

- Higher active modulators (inhibitors) and combinational recipes 


\section{Chemical or biological agents}

(Medicinal chemistry, chemical extracting methodology, analytic chemistry, new drug delivery systems

Tumor models

(Gene-knocked or modified, highly metastatic tumor, drug-resistant tumor, tumor cell line narrow-down, evaluation efficiency improvements etc)

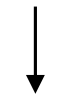

\section{Drug evaluation systems}

(Different sites of tumor inoculation, human tumor xenografts, efficiency of evaluation system, modern

$$
\text { analytical instruments etc) }
$$

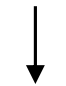

(Compound tolerance, ADME, toxicology reports, modern chromatography, pharmacogenomics etc)

\section{Preclinical and clinical evaluations}

(Modern diagnostics-related therapy, genome-wide-associate studies, cost-effective, personalized medicine etc)

Figure 1: Major pathways to update anticancer drug development chain.

against neoplasm metastasis and cancer stem cells [47-49] must be formal clinical cancer therapeutic agents and conventional cancer treatment options.

- Modifying tumor models for different therapeutic options other than chemical agents, such as biotherapy or immunotherapy. Biotherapy or immunotherapy has been emerging powers to combat and counteract cancer growth and metastases in clinical trials [46]. Nonetheless, they are inappropriate to be evaluated by conventional drug screening or verification systems for chemical compounds. Creative steps or new models must be innovated tailored for evaluations of biotherapy and so on.

- Genetic modifying animal tumor models to make quicker obtaining useful information about therapeutic efficacies and outcomes in clinics must be established in anticancer drug screening and preclinical studies.

- Testify more natural chemical compounds because greater portions of highly active anticancer drugs are natural-borne drugs [50,51]. By taking this avenue, we can possibly find more effective anticancer drugs.

\section{“Drug” toxicity evaluations}

Evaluations of compound toxicities can be through many different routes. A lot of money must be paid for evaluations of different levels of drug toxicology. Unfortunately, it is a work of multi-disciplinary. Apart from discovery of drug toxicological mechanisms of action and literature publications in international journals, balancing the expenditures of all branches of drug discovery, development, toxicology and licensing parameters is an ingenious enterprise because experimental or clinical "drug" toxicity evaluations are limitless. From our personal opinions, many repeat or copy of mechanisms of action from previous licensed anticancer drug study is low quality. Some tested compounds with low cytotoxicity against primary tumors might be highly effective antimetastatic activities. It is not a waste of time and money because a great number of anticancer compounds may never be licensed owing to their poor performance in primary cancer treatments. Yet, high quality study on specific mechanisms of potential targets and toxicities in most widely-used drugs is invaluable.

\section{Future Direction}

Facing these dilemma in drug developments, some personal perspectives are given. They are included: 
- Drug activity or toxicity studies vs drug mechanism studies; only original scientific investigations are most valuable;

- Modernizing experimental cancer models-including conventional protocols, genetic modified animals and human gene-knockout/ modified tumor cell lines and drug developmental pipelines updating;

- Reshuffle the present mode of drug manufacturing and marketing systems. Certainly, good manufacture practice (GMP) should be always adhered in all processes of drug development and manufactures;

- Strictly monitor money spending across each stage of drug development and pre-clinical treatment studies. Only following these efforts, we can ease current vicious circle of anticancer drug development and manufactures-skyrocketing new anticancer drug fee in clinical cancer trials;

- Find out the relationship between disease pathogenesis and drug therapeutic efficacies from human genomic study by cutting-edge techniques, such as bioinformatics and next generation sequencing (NGS) [51-53].

- Finding new mechanisms of action and modulators (inhibitors) of cancer stem cells [47-49] and drug combinations;

- Implementing and innovating procedures and strategies of PCT in experimental studies and clinical applications [40-44].

- Strong cooperation among different areas of research institutes, universities, drug manufacture companies and countries.

\section{Conclusion}

Owing to the slow progresses of anticancer drug development chain, rethink and retrospect past experience is quite necessary. In future, several future avenues might be gone through; (i) promote the efficacy of drug screening processes; (ii) find out more important drug targets; (iii) optimally control drug development expenses;(iv) hire highly talented personnel in drug developments. These are important topics and subjects for drug developments and manufactures internationally. In future, higher efficient experimental tumor growth or metastasis models and good governmental regulatory measures must be implemented by modernizing lab facilities and finding relevance good clinical paradigms worldwide. We look forward to a new era of upcoming anticancer drug discovery and developments.

\section{Acknowledgements}

This work was funded by Shanghai Science and Technology Foundation of High Educations 97A49.

\section{Competing Interests}

Authors have declared that no competing interests exist.

\section{References}

1. Siegel RL, Miller KD, Jemal A (2015) Cancer statistics, 2015. CA Cancer J Clin 65: 5-29.

2. Ali I, Rahis-ud-din SK, Aboul-Enein HY, Rather A (2011) Social aspects of cancer genesis. Cancer Ther 8: 6-14.

3. Mina LA, Sledge GW Jr (2011) Rethinking the metastatic cascade as a therapeutic target. Nat Rev Clin Oncol 8: 325-332.

4. Lu DY, Lu TR (2010) Antimetastatic activities and mechanisms of bisdioxopiperazine compounds. Anti-Cancer Agents in Medicinal Chemistry (Formerly Current Medicinal Chemistry-Anti-Cancer Agents) 10: 564-570.
5. Lu DY, Lu TR, Cao S (2012) Cancer metastases and clinical therapies. Cell Dev Biol 1: e110.

6. Lu DY, Lu TR, Wu HY, Cao S (2013) Cancer Metastasis treatments. Current Drug Therapy 8: 24-29.

7. Lu DY, Lu TR, Xu B, Qi RX, Yarla N, et al. (2016) Cancer Metastasis, a Clinical Dilemma for Therapeutics. Current Drug Therapy 11: 163-169.

8. Suggitt M, Bibby MC (2005) 50 years of preclinical anticancer drug screening: empirical to target-driven approaches. Clin Cancer Res 11: 971-981.

9. Talmadge JE, Singh RK, Fidler IJ, Raz A (2007) Murine models to evaluate nove and conventional therapeutic strategies for cancer. Am J Pathol 170: 793-804.

10. Lu DY, Chen EH, Lu TR (2015) Anticancer drug development, a matter of money or a matter of idea. Metabolomics 5: e134.

11. Mervis J (2005) Productivity counts - but the definition is key. Science 309: 726

12. Gupta SC, Sung B, Prasad S, Webb LJ, Aggarwal BB (2013) Cancer drug discovery by repurposing: teaching new tricks to old dogs. Trends Pharmacol Sci 34: 508-517.

13. Ruggeri BA, Camp F, Miknyoczki S (2014) Animal models of disease: preclinical animal models of cancer and their applications and utility in drug discovery. Biochemical pharmacology 87: 150-161.

14. Fidler IJ (1990) Critical factors in the biology of human cancer metastasis: Twenty-eight GHA Clowes memorial awards lecture. Cancer Res 50: 61306138.

15. Fidler IJ (2003) The pathogenesis of cancer metastasis: the 'seed and soil' hypothesis revisited. Nat Rev Cancer 3: 453-458.

16. Gupta GP, Massagué J (2006) Cancer metastasis: building a framework. Cell 127: 679-695.

17. Talmadge JE, Fidler IJ (2010) AACR centennial series: the biology of cancer metastasis: historical perspective. Cancer Res 70: 5649-5669.

18. Valastyan S, Weinberg RA (2011) Tumor metastasis: molecular insights and evolving paradigms. Cell 147: 275-292.

19. Herter-Sprie GS, Kung AL, Wong KK (2013) New cast for a new era: preclinical cancer drug development revisited. J Clin Invest 123: 3639-3645.

20. Sava G, Bergamo A (1999) Drug control of solid tumour metastases: a critica view. Anticancer Res 19: 1117-1124.

21. Kessenbrock K, Plaks V, Werb Z (2010) Matrix metalloproteinases: regulators of the tumor microenvironment. Cell 141: 52-67.

22. Taraboletti G, Margosio B (2001) Antiangiogenic and antivascular therapy for cancer. Curr Opin Pharmacol 1: 378-384.

23. Schein PS, Scheffler B (2006) Barriers to efficient development of cance therapeutics. Clin Cancer Res 12: 3243-3248.

24. Span PN (2015) From eels to the importance of cancer biobanks. Future Sc OA 1: FSO65.

25. Ali I, Wani W, Saleem K, Haque A (2012) Thalidomide: A banned drug resurged into future anticancer drug. Current Drug Therapy 7: 13-23.

26. Lu DY, Lu TR (2015) Mathematics or physics-majored students on the biomedical fields, insiders or outsiders. Metabolomics 5: e142.

27. Lu DY, Zhu PP, Wu HY, Yarla NS, Zhu H (2016) Human Suicide Study, is there an Association between Suicide and Mental Illness. Metabolomics (Los Angel) 6: 2153-0769.

28. Lu DY, Chen EH, Wu HY, Lu TR, Xu B, et al. (2017) Anticancer drug combinations, how far we can go through?. Anti-Cancer Agents in Medicinal Chemistry (Formerly Current Medicinal Chemistry-Anti-Cancer Agents) 17: 21-28.

29. Lu DY, Chen EH, Lu TR, Ding J (2015) Anticancer drug combinations, studies from different pathways. Cell Dev Biol 4: 2 .

30. Lu DY, Chen EH, Lu TR, Ding J (2016) Anticancer drug combinations, studies for all possibilities. Advances in Pharmacoepidemiology \& Drug Safety 5: e138.

31. Ali I (2011) Nano anti-cancer drugs: pros and cons and future perspectives. Curr Cancer Drug Targets 11: 131-134.

32. Ali I, Rahis-Uddin R, Salim K, Rather MA, Wani WA, et al. (2011) Advances in nano drugs for cancer chemotherapy. Curr Cancer Drug Targets 11: 135-146. 
33. Ugurel S, Schadendorf D, Pföhler C, Neuber K, Thoelke A, et al. (2006) In vitro drug sensitivity predicts response and survival after individualized sensitivitydirected chemotherapy in metastatic melanoma: a multicenter phase II trial of the Dermatologic Cooperative Oncology Group. Clinical cancer research 12 : 5454-5463.

34. Lu DY, Lu TR, Ding J, Xu B, Che JY, et al. (2015) Anticancer drug sensitivity testing, a historical review and future perspectives. Current Drug Therapy 10: 44-55.

35. Volm M, Efferth T (2015) Prediction of Cancer Drug Resistance and Implications for Personalized Medicine. Front Oncol 5: 282

36. Huang RS, Ratain MJ (2009) Pharmacogenetics and pharmacogenomics of anticancer agents. CA Cancer J Clin 59: 42-55.

37. Patel JN, Fuchs CS, Owzar K, Chen Z, McLeod HL (2013) Gastric cance pharmacogenetics: progress or old tripe? Pharmacogenomics 14: 1053-1064.

38. André F, Ciccolini J, Spano JP, Penault-Llorca F, Mounier N, et al. (2013) Personalized medicine in oncology: where have we come from and where are we going? Pharmacogenomics 14: 931-939.

39. Lu DY, Lu TR, Xu B, Ding J (2015) Pharmacogenetics of cancer therapy: breakthroughs from beyond? Future Sci OA 1: FSO80.

40. Lu DY, Chen XL, Ding J (2006) Individualized cancer chemotherapy integrating drug sensitivity tests, pathological profile analysis and computational coordination - an effective strategy to improve clinical treatment. Med Hypotheses 66: 45-51.

41. Lu DY, Lu TR, Chen XL, Ding J (2012) Individualized cancer chemotherapy. Hypotheses in Clinical Medicine. In: Shoja MM, Agutter PS, Tubbs RS, Ghanei M, Ghabili K, et al. Nova Science Publisher. USA 13: 199-216.

42. Lu DY, Lu TR, Wu HY (2014) Personalized cancer therapy, a perspective. Clin Exp Pharmacol 4: 153
43. Lu DY, Lu TR, Che JY, Wu HY (2014) Individualized cancer therapy. Innovations in Pharmaceuticals and Pharmacotherapy 2: 414-425.

44. Lu DY (2014) Personalized cancer chemotherapy, an effective way for enhancing outcomes in clinics. Woodhead Publishing, Elsevier, UK.

45. Couzin-Frankel J (2013) Breakthrough of the year 2013. Cancer immunotherapy Science 342: 1432-1433.

46. Lu DY, Lu TR, Wu HY (2013) Cost-effectiveness considerations of individualized cancer chemotherapy. Advances in Pharmacoepidemiology \& Drug Safety 2 e121.

47. Yakisich JS (2012) Challenges and limitations of targeting cancer stem cells and/or the tumour microenvironment. Drug and Therapy Study 2: e10.

48. Park TS, Donnenberg VS, Donnenberg AD, Zambidis ET, Zimmerlin L (2014) Dynamic interactions between cancer stem cells and their stromal partners. Current Pathology Reports 2: 41-52.

49. Magee JA, Piskounova E, Morrison SJ (2012) Cancer stem cells: impact heterogeneity, and uncertainty. Cancer Cell 21: 283-296.

50. Ali I, Saleem K, Uddin R, Haque A, El-Azzouny A (2010) Natural products: human friendly anti-cancer medications. Egypt Pharm J (NRC) 9: 133-179.

51. Lu DY, Lu TR, Lu Y, Sastry N, Wu HY (2016) Discover natural chemical drugs in modern medicines. Metabolomics 6: 181.

52. Lander ES (2011) Initial impact of the sequencing of the human genome Nature 470: 187-197.

53. Braggio E, Egan JB, Fonseca R, Stewart AK (2013) Lessons from nextgeneration sequencing analysis in hematological malignancies. Blood Cancer J 3: e127. 